MATEC Web of Conferences 51, 03001 (2016)

DOI: $10.1051 /$ matecconf/20165103001

(C) Owned by the authors, published by EDP Sciences, 2016

\title{
Experimental Investigation on the Performance of Grinding Assisted Electrochemical Discharge Drilling of Glass
}

\author{
Ladeesh V G ${ }^{a}$, Manu R \\ National Institute of Technology Calicut, Mechanical Engineering Department, Kerala- 673601, India
}

\begin{abstract}
Grinding assisted electrochemical discharge drilling (G-ECDD) is a novel technique for producing micro and macro holes in brittle materials including advanced ceramics and glass, both efficiently and economically. G-ECDD involves the use of a rotating diamond core drill as the tool in a normal electrochemical discharge machine setup. The material removal happens by a combination of thermal melting due to electric discharges, followed by grinding action of diamond grits and chemical etching action. In this study, the effect of process parameters like voltage, duty cycle, cycle time and electrolyte concentration on material removed (MR) was investigated systematically using response surface methodology. Analysis of variance was performed to identify the significant factors and their percentage contribution. The most significant factor was found to be duty cycle followed by voltage, cycle time and concentration. A quadratic mathematical model was developed to predict MR. Tool wear was found for different frequencies and voltages. Higher tool wear was observed for high frequency above $5 \mathrm{kHz}$ pulsed DC supply at high voltage of $110 \mathrm{~V}$. Tool wear at the end face of the tool was found to be a significant problem affecting the tool life.
\end{abstract}

\section{Introduction}

Grinding assisted electrochemical discharge drilling (G-ECDD) is a hybrid machining technique which utilizes the combined effect of electrochemical discharges, grinding action of diamond coated/impregnated tool along with high temperature chemical etching action of the electrolyte for removing material from the workpiece. Here, the rotating tool will be polarized as the cathode and a steel plate will be maintained as the anode. Both the electrodes are dipped in an electrolyte and a pulsed DC voltage will be applied between the electrodes. The electrochemical reactions at the cathode liberates hydrogen bubbles which will combine to form a gas film at the critical voltage and the film separates the tool from the electrolyte. This develops high current density at the tool tip and causes ionization of the hydrogen gas inside the film. An avalanche of electrons which appear as an electric arc strikes the workpiece surface placed at the vicinity of discharge. The kinetic energy of electrons will be dissipated in the form of heat causing the melting of the workpiece. The rotating tool with diamond grits at its tip remove the molten material from the machining zone. This reduces the heat affected zone and increase the MRR. Only few researchers have conducted a systematic study of ECDM process using abrasive tools and there is a great need to unveil the full potential of G-ECDD through proper research strategies.

\footnotetext{
${ }^{a}$ Corresponding author : ladeesh.vg@gmail.com
} 
Jain et al. [1] performed electrochemical discharge machining of alumina and glass using conventional cutting tool (CCT) and abrasive cutting tool (ACT). The material removed for different voltages and temperatures was found to be high for ACT when compared with CCT. Sanjay and Venkateswara [2] attempted drilling of alumina with a rotary abrasive electrode by ECDM. They found that the use of pulsed DC can improve the quality of the hole with a reduction in cracks. Liu et al. [3] used grinding aided ECDM for the machining of composites (alumina particles reinforced aluminium alloy) for the first time. They confirmed the material removal mechanism as a combination of ECDM and grinding action of the diamond core drill from SEM images. Sanjay and Venkateswara [4] made an attempt to make larger diameter hole in alumina using trepanning operation. An increase in the volume of material removed was observed with an increase in voltage, duty factor and conductivity of electrolyte. Peng and Liao [5] used traveling wire- ECDM for slicing glass rod and quartz bar. They found that frequency and duty ratio interact mutually and decides the energy density of the spark. A detailed study of current wave forms during ECDM was conducted and they identified two modes of current pulse (higher and smaller current impulses).

This study aims to reveal the performance of G-ECDD for machining glass and to find a practical solution to reduce the tool wear rate. A central composite design was used to develop the experimental runs. The machining parameters selected are voltage, duty cycle, cycle time and electrolyte concentration. A quadratic mathematical model was developed to predict material removed (MR). Additional experiments are performed to study the effect of voltage and frequency on tool wear rate.

\section{Experiment Details}

\subsection{Experimental setup}

All the experiments are performed using a modified CNC Router (Make- Newclear technologies, India: Model- TR 203) which is shown in Fig. 1. The experimental setup consist of an electrolyte bath which serves as the machining chamber, attachment for supplying power to rotating tool, a spring fed tool arrangement and a DC power supply. The spring fed tool arrangement was designed and fabricated so as to enable smooth feed of the tool and avoids the mechanical grinding action of diamond grits to predominate. This spring fed tool system ensures a proper balance of different material removal mechanisms in producing holes with minimal defects. A customized power supply capable of delivering pulsed DC with provisions for varying duty cycle and frequency was used for the experiments.

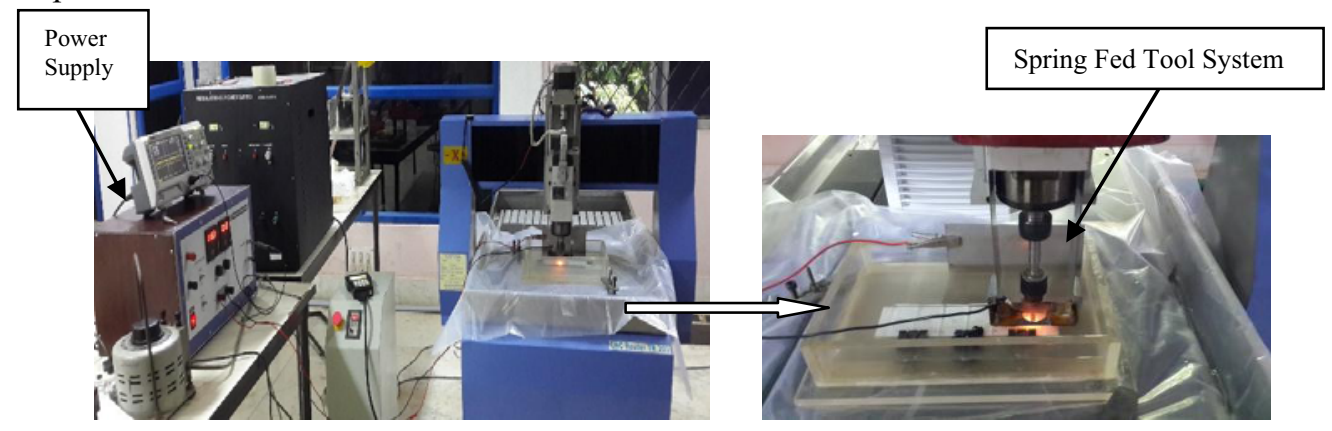

Figure 1. Integrated experimental setup for G-ECDD

The workpiece used was sodalime glass (Make- Saint gobain, India) of dimension 40(L) x 30(B) x $5(\mathrm{H}) \mathrm{mm}$. The electrolyte used was potassium hydroxide $(\mathrm{KOH})$. A thermostat with heating coil was used to maintain the temperature of electrolyte at $40^{\circ}$ Celsius. Material removed was determined by 
measuring the difference of the weight of workpiece before and after machining. Tool wear was also determined in a similar manner by taking the weight of the tool before and after machining.

\subsection{Design of experiment}

The central composite rotatable design (CCRD) has been adopted to study the four process parameters $(n=4)$. The CCRD design consist of $2^{n}$ factorial points, $2 n$ axial points and 7 centre points which gives a total of 31 runs. A design that produces contours of constant standard deviation of predicted response is called rotatable design. A central composite design can be made rotatable by choosing the $\alpha$ value of axial points equal to $n^{1 / 4}$. Here the number of factors being 4 , the value of $\alpha=2$. Hence the coded values for different levels in CCRD are $-2,-1,0,1,2$. The selected factors and their levels are shown in Table 1.

Analysis of variance (ANOVA) was conducted to identify the significant factors and the main interactions. ANOVA is a method of partitioning total variation into accountable sources of variation in an experiment. The technique can be used to interpret experimental data and make decision about the parameters under study

Table 1. Factors and their levels for CCRD

\begin{tabular}{|c|c|c|c|c|c|c|c|}
\hline \multirow{2}{*}{ Symbol } & Parameters & \multirow{2}{*}{ Units } & \multicolumn{5}{|c|}{ Coded levels } \\
\cline { 4 - 8 } & & & $\mathbf{- 2}$ & $\mathbf{- 1}$ & $\mathbf{0}$ & $\mathbf{1}$ & $\mathbf{2}$ \\
\hline $\mathrm{A}$ & Voltage & $\mathrm{V}$ & 90 & 95 & 100 & 105 & 110 \\
\hline $\mathrm{B}$ & Duty cycle & & 0.4 & 0.5 & 0.6 & 0.7 & 0.8 \\
\hline $\mathrm{C}$ & Cycle time & $\mathrm{s}$ & 0.0002 & 0.00065 & 0.00110 & 0.00155 & 0.002 \\
\hline $\mathrm{D}$ & Electrolyte ncentration & $\mathrm{M}$ & 2 & 2.5 & 3 & 3.5 & 4 \\
\hline
\end{tabular}

\section{Results and Discussion}

\subsection{Results of CCRD}

Experiments are conducted with central composite rotatable design and the results obtained are shown in Table 2. Randomization of the run order was performed for averaging out the effect of noise factors. A statistical software MINITAB-17 (trail version) was used for the analysis of the data. The main effects plot of the material removed is shown in Fig. 2. All the experiments were performed for a duration of two minutes and the weight of the workpiece before and after machining was taken to determine the material removed (MR).

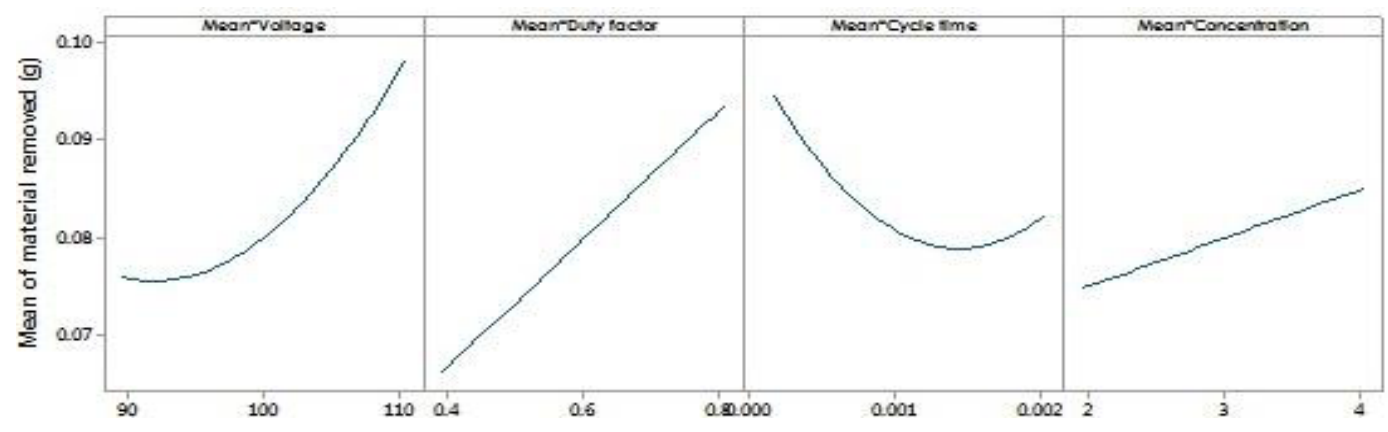

Figure 2. Main effects plot for material removed 
As voltage increases, the material removal also increases. An increase in voltage will produce discharges of higher energy. This high energy discharges cause the melting of workpiece surface in the form of craters. The crater volume will be more for discharges at higher voltage and this will increase the overall material removed.

Duty cycle can be defined as the ratio of pulse-on time to the total cycle time (pulse-on + pulse-off time). Hence a higher value of duty cycle indicates a higher duration of pulse supply time in a cycle. An increase in duty cycle will deliver the discharge energy for more duration and hence cause more material to melt. This accounts for the rise in material removed with an increase in duty cycle.

Cycle time decides the frequency of sparking (frequency $=1 /$ cycle time). At low cycle time, the frequency will be high and this produce large number of sparks at a particular location causing more material to be removed. When cycle time increases the frequency of sparking decreases causing less material removal.

Table 2. Results of CCRD

\begin{tabular}{|c|c|c|c|c|c|c|}
\hline Standard order & Run order & $\mathbf{A}$ & B & C & D & Material removed (g) \\
\hline 1 & 1 & 95 & 0.5 & 0.00065 & 2.5 & 0.0747 \\
\hline 2 & 6 & 105 & 0.5 & 0.00065 & 2.5 & 0.0857 \\
\hline 3 & 11 & 95 & 0.7 & 0.00065 & 2.5 & 0.0777 \\
\hline 4 & 18 & 105 & 0.7 & 0.00065 & 2.5 & 0.0887 \\
\hline 5 & 25 & 95 & 0.5 & 0.00155 & 2.5 & 0.0577 \\
\hline 6 & 30 & 105 & 0.5 & 0.00155 & 2.5 & 0.0788 \\
\hline 7 & 7 & 95 & 0.7 & 0.00155 & 2.5 & 0.0891 \\
\hline 8 & 19 & 105 & 0.7 & 0.00155 & 2.5 & 0.0918 \\
\hline 9 & 2 & 95 & 0.5 & 0.00065 & 3.5 & 0.0792 \\
\hline 10 & 12 & 105 & 0.5 & 0.00065 & 3.5 & 0.0902 \\
\hline 11 & 8 & 95 & 0.7 & 0.00065 & 3.5 & 0.0912 \\
\hline 12 & 26 & 105 & 0.7 & 0.00065 & 3.5 & 0.1032 \\
\hline 13 & 13 & 95 & 0.5 & 0.00155 & 3.5 & 0.0692 \\
\hline 14 & 20 & 105 & 0.5 & 0.00155 & 3.5 & 0.0812 \\
\hline 15 & 3 & 95 & 0.7 & 0.00155 & 3.5 & 0.0852 \\
\hline 16 & 27 & 105 & 0.7 & 0.00155 & 3.5 & 0.0962 \\
\hline 17 & 21 & 90 & 0.6 & 0.0011 & 3.0 & 0.0769 \\
\hline 18 & 14 & 110 & 0.6 & 0.0011 & 3.0 & 0.0948 \\
\hline 19 & 31 & 100 & 0.4 & 0.0011 & 3.0 & 0.0654 \\
\hline 20 & 9 & 100 & 0.8 & 0.0011 & 3.0 & 0.0908 \\
\hline 21 & 22 & 100 & 0.6 & 0.0002 & 3.0 & 0.0941 \\
\hline 22 & 28 & 100 & 0.6 & 0.002 & 3.0 & 0.0801 \\
\hline 23 & 15 & 100 & 0.6 & 0.0011 & 2.0 & 0.0775 \\
\hline 24 & 23 & 100 & 0.6 & 0.0011 & 4.0 & 0.0813 \\
\hline 25 & 4 & 100 & 0.6 & 0.0011 & 3.0 & 0.0801 \\
\hline 26 & 16 & 100 & 0.6 & 0.0011 & 3.0 & 0.0791 \\
\hline 27 & 10 & 100 & 0.6 & 0.0011 & 3.0 & 0.0793 \\
\hline 28 & 24 & 100 & 0.6 & 0.0011 & 3.0 & 0.0821 \\
\hline 29 & 29 & 100 & 0.6 & 0.0011 & 3.0 & 0.0843 \\
\hline 30 & 17 & 100 & 0.6 & 0.0011 & 3.0 & 0.0801 \\
\hline 31 & 5 & 100 & 0.6 & 0.0011 & 3.0 & 0.0771 \\
\hline
\end{tabular}

When concentration increases, the conductivity of the electrolyte increases and the electrochemical reaction is accelerated. This cause more number of hydrogen bubbles to be liberated at the tool surface and the gas film formation happens at a faster rate. This helps to utilize a greater portion of the energy supplied per pulse for arcing and hence the material removed will be more at higher electrolyte 
concentration. Thus the highest MR of $0.1032 \mathrm{~g}$ was obtained for the combination of high voltage of $105 \mathrm{~V}$, high duty cycle of 0.7 , low cycle time of $0.00065 \mathrm{~s}$ and high concentration of $3.5 \mathrm{M}$. The drilled holes using G-ECDD are shown in Fig. 3.

\subsection{ANOVA and regression analysis}

ANOVA results are given in Table 3. From the $F$-values, the most significant factor was found to be duty cycle followed by voltage, cycle time and concentration. The square effects of voltage and cycle time are found to be significant. The two- way interaction of duty cycle and cycle time was also found to be significant. A second order regression model was developed for material removed (MR) and is given by (1).

$\mathrm{MR}=0.624-0.01185^{*}$ Voltage $-0.0021 *$ Duty factor $-64.4 *$ Cycle time $+0.00493 *$ Concentration $+0.000065 *$ Voltage*Voltage $+9560 *$ Cycle time*Cycle time $+61.5 *$ Duty factor*Cycle time

Backward elimination regression was used to eliminate insignificant terms and make the model more efficient. The standard deviation of material removed is 0.00341 which is very less. The coefficient of multiple determination $\left(R^{2}\right)$ and adjusted $R^{2}$ for MR are 89.78 and 86.67 respectively which are high and indicate that the model terms are significant and the model can be used for predicting the response. Lack-of-fit was found to be insignificant which means that the model can fit the data well.

Table 3. ANOVA table for material removed

\begin{tabular}{|l|c|c|c|c|c|c|}
\hline Source & Sum of Squares & $\begin{array}{c}\text { Degrees of } \\
\text { freedom }\end{array}$ & $\begin{array}{c}\text { Mean } \\
\text { Square }\end{array}$ & $\begin{array}{c}\mathbf{F} \\
\text { Value }\end{array}$ & $\begin{array}{c}\text { p-value } \\
\text { Prob > F }\end{array}$ & $\begin{array}{c}\text { Percentage } \\
\text { Contribution (\%) }\end{array}$ \\
\hline Model & 0.0023508 & 7 & 0.0003358 & 28.868271 & $<0.0001$ & \\
\hline A & 0.0006813 & 1 & 0.0006813 & 58.567876 & $<0.0001$ & 26 \\
\hline B & 0.00103 & 1 & 0.00103 & 88.540853 & $<0.0001$ & 39 \\
\hline C & 0.0002003 & 1 & 0.0002003 & 17.219183 & 0.0004 & 8 \\
\hline D & 0.0001458 & 1 & 0.0001458 & 12.534083 & 0.0017 & 6 \\
\hline BC & 0.0001226 & 1 & 0.0001226 & 10.536101 & 0.0036 & 4.6 \\
\hline$A^{*}$ A & $7.605 \mathrm{E}-05$ & 1 & $7.605 \mathrm{E}-05$ & 6.5375305 & 0.0176 & 2.9 \\
\hline C*C & 0.0001094 & 1 & 0.0001094 & 9.4049748 & 0.0055 & 4.1 \\
\hline Residual & 0.0002676 & 23 & $1.163 \mathrm{E}-05$ & & & \\
\hline Lack of Fit & 0.0002355 & 17 & $1.386 \mathrm{E}-05$ & 2.596983 & 0.1215 & \\
\hline Pure Error & $3.201 \mathrm{E}-05$ & 6 & $5.335 \mathrm{E}-06$ & & & \\
\hline Cor Total & 0.0026183 & 30 & & & & \\
\hline
\end{tabular}

\subsection{Effect of voltage and frequency on tool wear}

The effect of voltage and pulse frequency on tool wear is shown in Fig. 4. An increase in voltage will deliver more current to the machining zone and causes ohmic heating of the tool and surrounding electrolyte. This local rise in temperature may deteriorate the bonding of diamond grits to the tool shank thus causing dislodging of grits and increase tool wear. The discharges occur randomly at the location of high electric field and the current flows to the discharge channel via the tool surface. At high frequency, the electric and magnetic field interacts. The sum of main current and eddy current rises on the electrode surface and the discharge probability from tool edge will be accelerated. This cause an increase in tool wear at the end face while using high frequency power supply when compared to low frequency. 


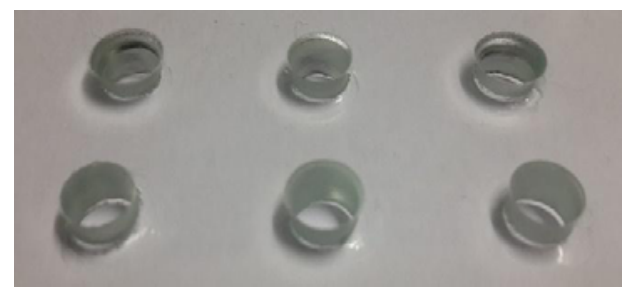

Figure 3. Machined holes of $6 \mathrm{~mm}$ diameter

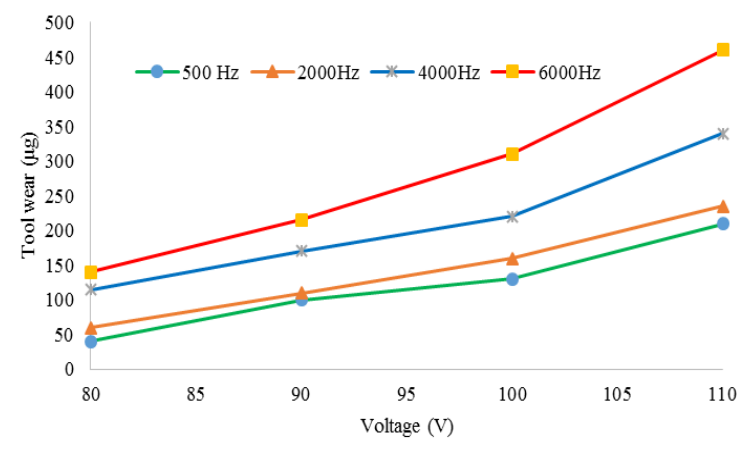

Figure 4. Effect of voltage and frequency on tool wear

\section{Conclusions}

The following conclusions are drawn from the above studies.

- Duty cycle and voltage are found to be the most significant factors that influence material removal. Cycle time and electrolyte concentration are the other main factors.

- The highest MR of $0.1032 \mathrm{~g}$ was obtained for the combination of high voltage (105V), high duty cycle $(0.7)$, low cycle time $(0.00065 \mathrm{~s})$ and high concentration of $3.5 \mathrm{M}$.

- The use of a low frequency $(500 \mathrm{~Hz})$ and low voltage $(80 \mathrm{~V})$ can significantly reduce the tool wear. The tool wear at the end face of the diamond core drill was found to be more predominant than the wear at the lateral face. Hence an increase in the thickness of diamond coating layer at the end face of the tool will increase the tool life.

\section{References}

1. V. K. Jain et al.," On the machining of alumina and glass," Int. J. of Mach. Tools \& Manuf. 42, 1269-1276 (2002).

2. K. Sanjay et al., "The drilling of Al2O3 using a pulsed DC supply with a rotary abrasive electrode by the electrochemical discharge process," Int. J. Adv. Manuf. Technol. 39, 633-641 (2008).

3. J. W. Liu et al., "Grinding-aided electrochemical discharge machining of particulate reinforced metal matrix composites," Int. J. Adv. Manuf. Technol. 68, 2349-2357 (2013).

4. K. Sanjay et al., "Trepanning of Al2O3 by electro-chemical discharge machining (ECDM)," Int. J. of Mach. Tools \& Manuf. 47, 2061-2070 (2007).

5. W. Y. Peng et al.,"Study of electrochemical discharge machining technology for slicing nonconductive brittle materials," J. of Mat. Pro. Tech. 149, 363-369 (2004). 